\title{
Approach to the Highest HXR Yield in Plasma Focus Device Using Adaptive Neurofuzzy Inference System to Optimize Anode Configuration
}

\author{
M. Mahtab, ${ }^{1}$ M. Taghipour, ${ }^{2}$ G. H. Roshani, ${ }^{3,4}$ and M. Habibi ${ }^{1}$ \\ ${ }^{1}$ Energy Engineering and Physics Department, Amirkabir University of Technology, Tehran, Iran \\ ${ }^{2}$ Department of Biomedical Engineering, Faculty of Medicine, Kermanshah University of Medical Sciences, Kermanshah, Iran \\ ${ }^{3}$ Faculty of Energy, Kermanshah University of Technology, Kermanshah, Iran \\ ${ }^{4}$ Young Researchers and Elite Club, Kermanshah Branch, Islamic Azad University, Kermanshah, Iran
}

Correspondence should be addressed to M. Mahtab; m.mahtab.83@gmail.com

Received 28 January 2014; Accepted 10 May 2014; Published 24 June 2014

Academic Editor: Tatyana Sizyuk

Copyright (C) 2014 M. Mahtab et al. This is an open access article distributed under the Creative Commons Attribution License, which permits unrestricted use, distribution, and reproduction in any medium, provided the original work is properly cited.

\begin{abstract}
Adaptive neurofuzzy inference system (ANFIS) is investigated to optimize the configuration of anode shape in plasma focus devices to achieve the highest X-ray yield. Variables of discharge voltage, filling gas pressure, and angles of anode slopes $\left(\Phi_{1}\right.$ and $\left.\Phi_{2}\right)$ are chosen as input parameters, while the output is designated to be the radiated hard X-ray intensity. The trained ANFIS has achieved good agreement with the experimental results and has mean relative error percentages (MRE\%) 1.12\% and 2.18\% for training and testing data, respectively. The study demonstrates that adaptive neurofuzzy inference system is useful, reliable, and low-cost way to interpret the highest $\mathrm{X}$-ray yield and corresponding anode configuration in plasma focus devices.
\end{abstract}

\section{Introduction}

Plasma foci are generally known as efficient, nonexpensive source of various pulsed radiations of high intensity including $\mathrm{X}$-rays [1, 2], ions [3], electrons [4], and neutrons [5]. The onset of radiations coincides with compression of filling gas into pinched plasma which is observed by significant fall in the current signal. Occurrence of instabilities, which compress plasma volume, leads to induced electric field and local micron-sized plasma regions (hot spots) of high density and temperature. Apart from possibility of fusion reactions in the case of deuterium plasma, hot spots are identified as sources of intense X-ray emission as well as ion beams and pulsed electrons [6, 7]. Accelerated electrons often have high energies in excess of the quantity which would be imparted on by the applied voltage. Electron beams mainly hit the central metallic electrode and slightly the chamber's wall emitting braking X-ray radiation of extensive continuous spectrum from soft up to hard X-ray on which characteristic lines represent the anode's material mount.
Various applications have been exploited from plasma foci's radiations especially due to characteristics of reproducibility, compact size, and cheapness. Ion beams of desired gas are generally used for surface treatments $[8,9]$ as well as neutron beams for neutronic detection of hydrogen in samples $[10,11]$ and lithography by means of electron beams $[12,13]$. X-rays, in particular, have extensive applications such as X-ray microscopy, X-ray lithography, micromachining, radiography, and material synthesis [14-21]. Radiography of the biological samples and X-ray imaging of moving metallic objects are two of the most noticeable applications of radiated hard X-ray in PF devices. HXR radiography of the biological samples $[18,22]$ which needs inevitable source characteristics, that is, intense emission through small dimension source and short exposure of time, is well fulfilled in PF devices. Moreover, extremely short duration of the X-ray emission (exposure time of about $50 \mathrm{~ns}$ ) together with the degree of penetration contributes to producing high quality X-ray images of moving metallic objects [23].

All PF devices, which were first constructed in two slightly different designs [24, 25], almost have focused plasma 
characteristics in common. However, focused plasma and resulting beams are highly affected by basic design parameters, such as dimensions and appropriate capacitor bank, electrodes, and chamber materials, as well as working conditions of operating phase such as mixture and pressure of filling gas. Comprehensive investigations have been devoted to study and optimize PF device emissions among which anode tip configuration is one of the highly effective ones [26, 27]. Most of performed studies have shown that modified anode tips, in which the anode diameter reduces at the end, are capable of increasing the X-ray yield about orders of magnitude. The modified anode tips enhance X-ray emission through significant increase in radiation time which resulted from consistent transition of current sheath from axial acceleration phase to radial implosion phase. Moreover, these configurations enhance changes in plasma column inductance which accelerate electron beams toward the anode end. The effects of modified anode tips configurations on current sheath motion can be found in [28] in detail. Although most of the investigations agree that the conical-based anode tips would be the best configuration for increasing emission yields, there has been no idea for the exact geometry which would lead to the highest X-ray yield thus far.

In this research, knowing the fact that the X-ray intensity varies by the anode shape, we focused our attention to find out the best configuration of the conical anode to achieve the highest hard X-ray yield. For this purpose, anode configuration is designed by two angles of $\Phi_{1}$ and $\Phi_{2}$ representing the height and degree of slope. The variables of $\Phi_{1}$ and $\Phi_{2}$ are assumed as input parameters of ANFIS together with discharge voltage and filling gas pressure; hence, the results of ANFIS obtain the highest HXR yield which is desirable for relative applications.

\section{Experimental Setup and Diagnostics}

The primary experimental tests are carried out by APF device [29] which is powered by a single $V_{0}=15 \mathrm{kV}, C_{0}=$ $40 \mu \mathrm{F}$, and $L_{0}=30 \mathrm{nH}$ capacitor with a total inductance of $110 \mathrm{nH}$. The inner electrode is a copper cylinder with a diameter and length of 20 and $148 \mathrm{~mm}$, respectively. The outer electrodes, which are composed of six copper rods, are designed with inner diameter of $44 \mathrm{~mm}$. Furthermore, electrodes are separated by a Pyrex tube of $48 \mathrm{~mm}$ length. The schematic of APF facility and assembled diagnostics is shown in Figure 1.

A Rogowski coil was used to record the current waveform and to ensure efficient energy transfer. The typical peak current and rise time of APF facility are shown in Figure 2. A fast plastic scintillation detector (NE102A, a $5 \mathrm{~cm}$ long, $5 \mathrm{~cm}$ diameter cylinder), coupled with a high gain photomultiplier tube (PM-53) biased at $-1.2 \mathrm{kV}$, located at a distance of $1 \mathrm{~m}$ from the window is used for the time resolved HXR measurement, while the photon's transition threshold of the glass window is about $20 \mathrm{keV}$. The typical recorded signal is also shown in Figure 3.

Primary experimental materials on HXR intensities are obtained by assembling three configurations of anode tips.

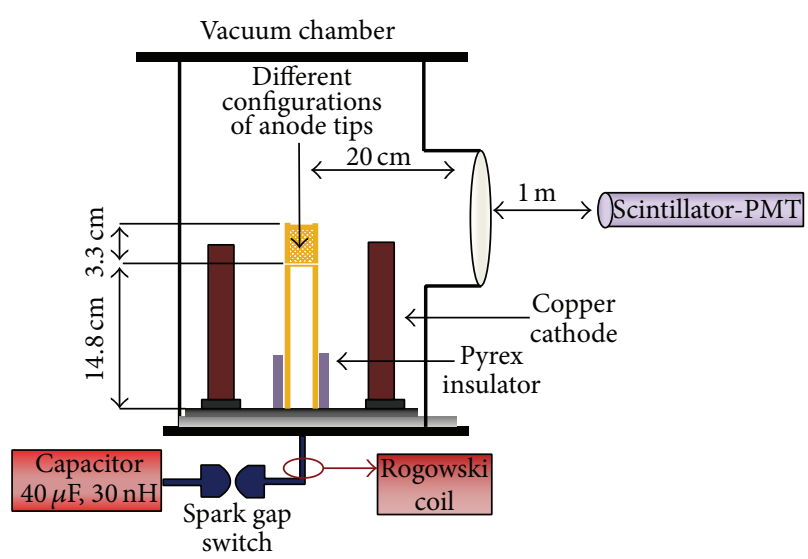

FIgUrE 1: The schematic of APF Mather-type plasma focus, electrodes, and the diagnostics.

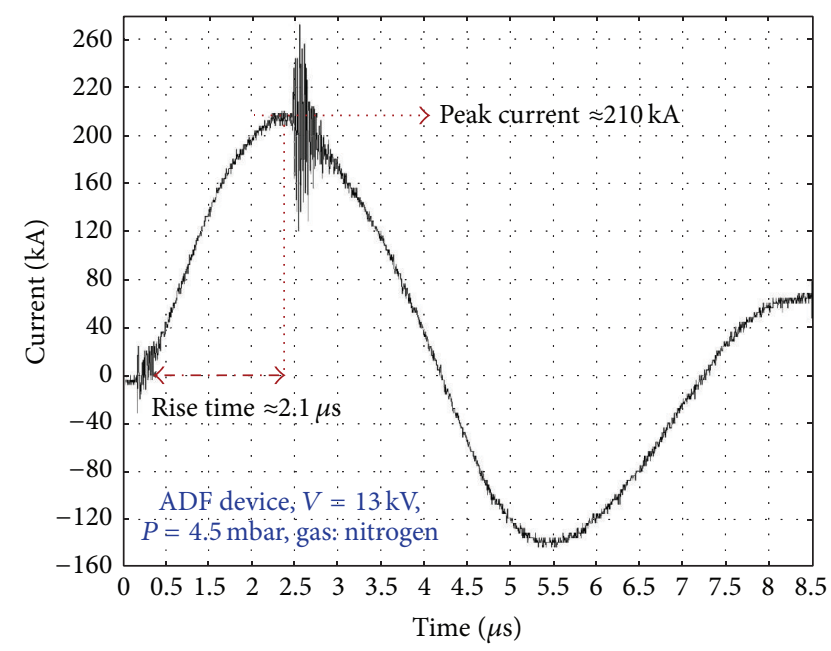

Figure 2: Typical Rogowski coil signal.

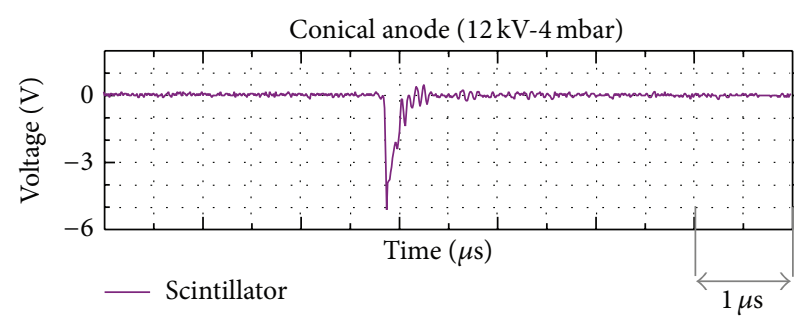

FIGURE 3: Typical signals of scintillator.

Tested configurations are cylindrical with flat top $\left(\Phi_{1}=\Phi_{2}=\right.$ $\left.90^{\circ}\right)$ and two conical anodes with different slopes $\left(\Phi_{1}=\right.$ $\Phi_{2}=79.7^{\circ}$ and $\Phi_{1}=90, \Phi_{2}=84.28^{\circ}$ ) which are shown, respectively, in Figure 4 from left to right. It should also be mentioned that while the total length of the anode is $14.8 \mathrm{~cm}$, the variable length of all tips is $3.3 \mathrm{~cm}$.

The experiments were done using nitrogen gas, scanning all practical pressures, while each pressure is tested successfully for 5 times. Averaged areas under the recorded scintillator signals between shots are represented as hard X-ray 


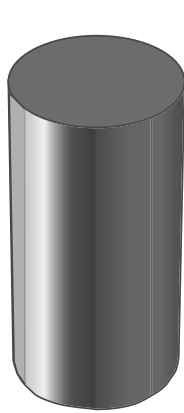

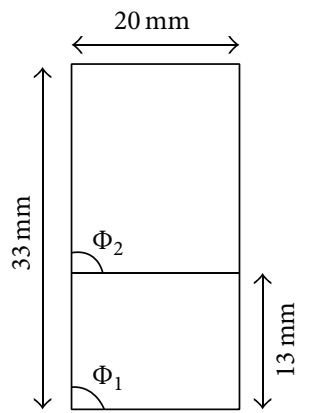

(a)
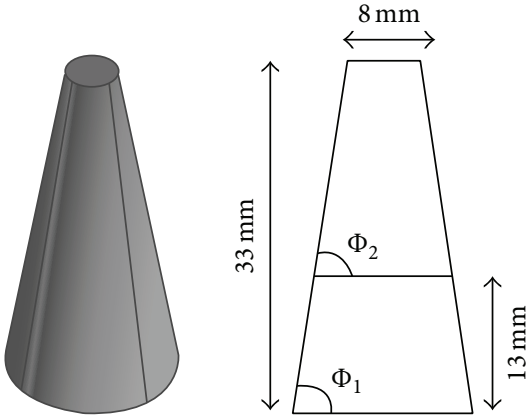

(b)

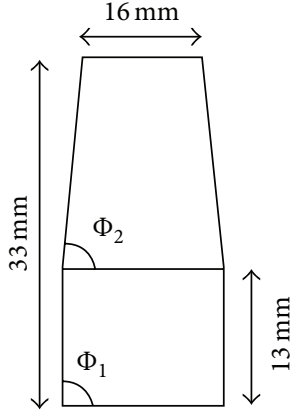

(c)

Figure 4: Three different configurations of anode tips.

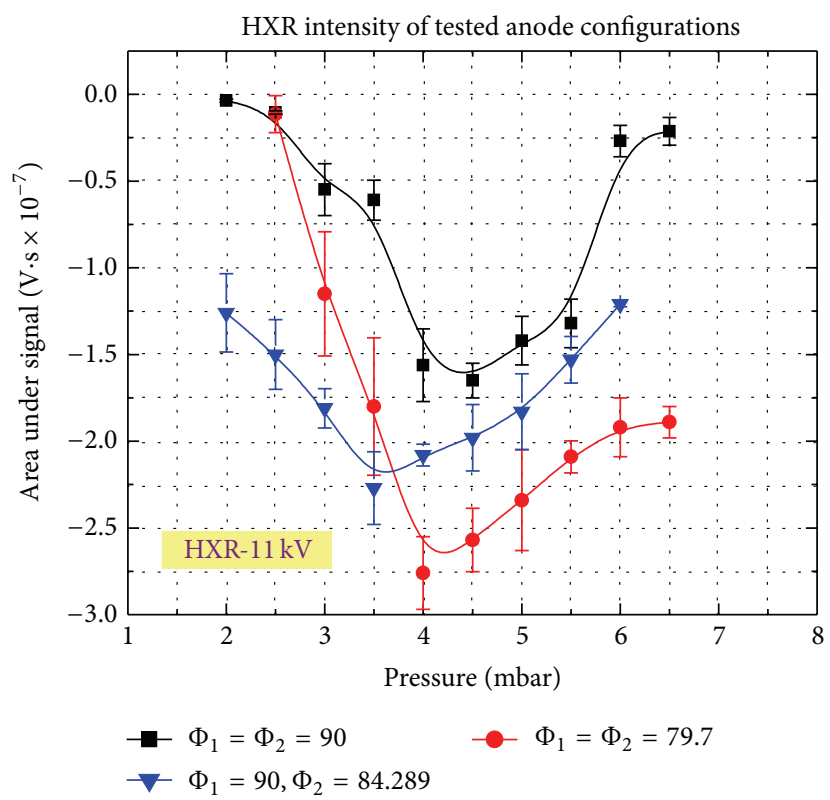

FIGURE 5: Dependency of HXR intensity versus filling pressure for different anode tips.

intensity to consider both time and voltage expansions. The peak detection and integration of the data were performed by using the SAP-ALCOR 9.20 software which has been developed in AmirKabir Fusion Laboratory [30]. The typical results of HXR measurement in discharge voltage of $11 \mathrm{kV}$ are shown in Figure 5.

\section{Optimization Approach}

Adaptive neurofuzzy inference system (ANFIS) is a multilayer feed-forward network, which uses artificial neural network (ANN) learning algorithms and fuzzy inference system (FIS) reasoning to map an input space to an output space [31]. The accurate method based on ANFIS is presented in order to achieve the highest X-ray yield in plasma focus device. The ANFIS structure of calculation is shown in Figure 6, where the inputs are discharge voltage (kilo Volt), working gas pressure (Torr), and anode design slopes of $\Phi_{1}$ and $\Phi_{2}$ (degree), while the output is assigned to be hard Xray intensity (Volt $\times$ second).
Description of the layers in the proposed ANFIS model is defined as follows $[32,33]$.

Layer 1. The node functions of this layer are given by (1), where $V, P, \Phi_{1}$, and $\Phi_{2}$ are the inputs of the model and $\mu_{A}(V), \mu_{B}(P), \mu_{C}\left(\Phi_{1}\right)$, and $\mu_{D}\left(\Phi_{2}\right)$ are the fuzzy membership functions:

$$
\begin{aligned}
A_{i}(V) & =\mu_{A i}(V), \quad i=1,2, \ldots, 27, \\
B_{i}(P) & =\mu_{B i}(P), \quad i=1,2, \ldots, 27, \\
C_{i}\left(\Phi_{1}\right) & =\mu_{C i}\left(\Phi_{1}\right), \quad i=1,2, \ldots, 27, \\
D_{i}\left(\Phi_{2}\right) & =\mu_{D i}\left(\Phi_{2}\right), \quad i=1,2, \ldots, 27 .
\end{aligned}
$$

Gaussian membership function is a typical membership function and is given by

$$
G(x)_{i}=\exp \left(-\frac{0.5\left(x-a_{i}\right)^{2}}{b_{i}^{2}}\right)
$$




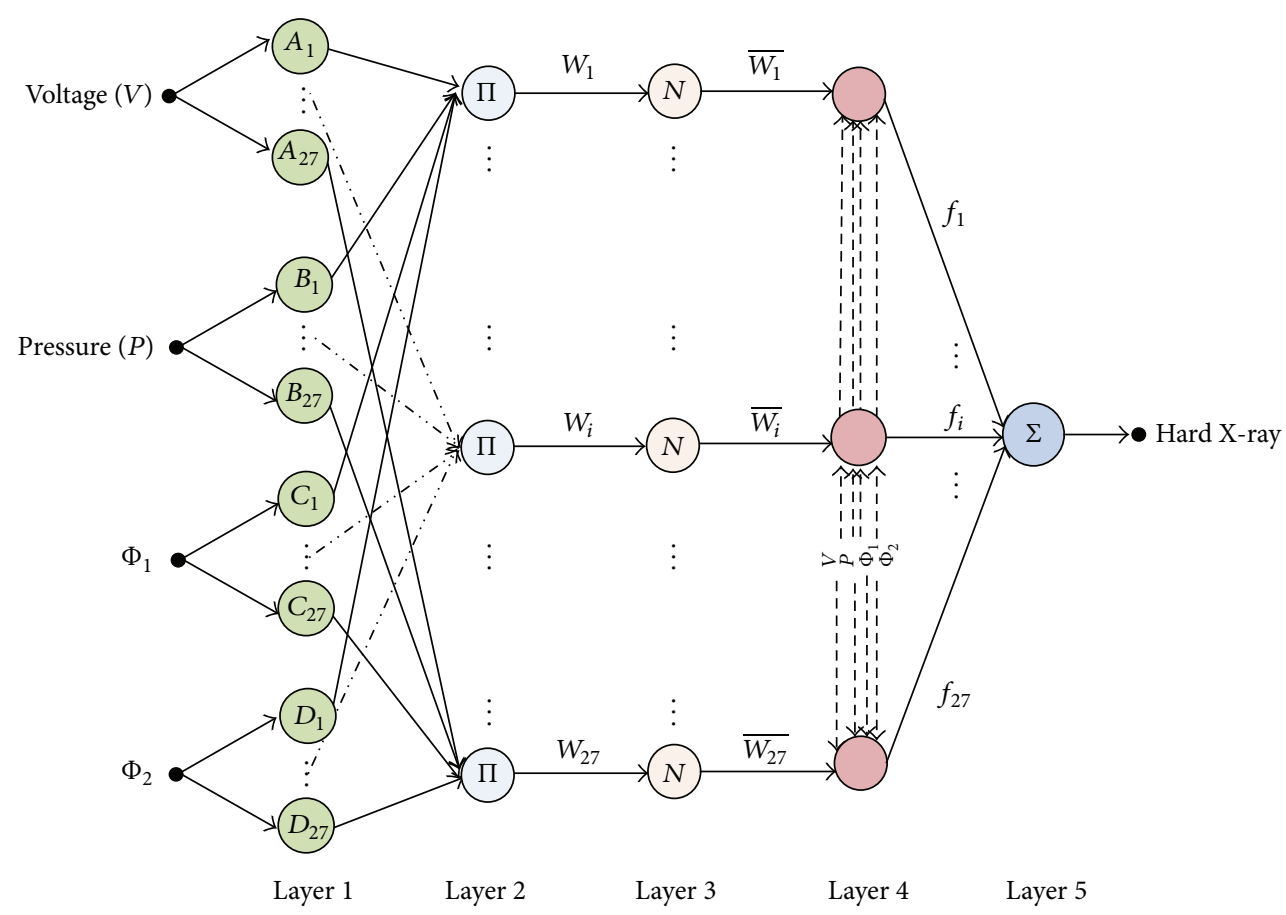

FIgURE 6: Proposed ANFIS structure.

where $a_{i}$ and $b_{i}$ are called premise parameters (nonlinear parameters).

Layer 2. The output of this layer is given by

$W_{i}=A_{i}(V) \times B_{i}(P) \times C_{i}\left(\Phi_{1}\right) \times D_{i}\left(\Phi_{2}\right), \quad i=1,2, \ldots, 27$.

Layer 3. This layer estimates the ratio of the $i$ th rule's firing strengths to the sum of all rule's firing strengths as follows:

$$
\bar{w}_{i}=\frac{w_{i}}{\sum_{j=1}^{27} w_{j}}, \quad i=1,2, \ldots, 27 .
$$

Layer 4 . The output of this layer is given by

$$
f_{i}=\bar{w}_{i}\left(m_{i} V+n_{i} P+r_{i} \Phi_{1}+l_{i} \Phi_{2}+k_{i}\right), \quad i=1,2, \ldots, 27,
$$

where $m_{i}, n_{i}, r_{i}, l_{i}$, and $k_{i}$ are called consequent parameters (linear parameters).

Layer 5. The output of this layer is given by

$$
\text { Hard X-Ray }=\sum_{i=1}^{27} f_{i} \text {. }
$$

The data sets required for training and testing the proposed ANFIS model were extracted from experimental materials. The whole data sets consisting of 75 data points were divided into two parts randomly which are (1) a training
TABLE 1: Specifications of the proposed ANFIS.

\begin{tabular}{lc}
\hline Type & Sugeno \\
Inputs/outputs & $4 / 1$ \\
Number of input membership functions & 27 for each input \\
Number of output membership functions & 27 \\
Input membership function type & Gaussian \\
Output membership function type & Linear \\
Number of fuzzy rules & 27 \\
Number of nonlinear parameters & 432 \\
Number of linear parameters & 135 \\
Number of epochs & 200 \\
\hline
\end{tabular}

or calibration set consisting of 52 data points (about 70\%) and (2) a validation or testing set consisting of 23 data points (about 30\%). Behavior of the system is recognized from the training set and validation of the behavior is examined using testing set. Consequently, the training set must be greater than testing one since there is necessity to use more data in order to train the network. In this study, different ANFIS structures were tested to obtain the best ANFIS configuration. Table 1 shows the specification of the best proposed ANFIS.

\section{Results and Discussion}

The comparisons between the experimental and interpreted results using the proposed ANFIS network, for training and testing data, are shown in Figures 7(a) and 7(b), respectively. Figure 7 is a regression diagram that shows the precision of calculations. The experimental values are those data which 


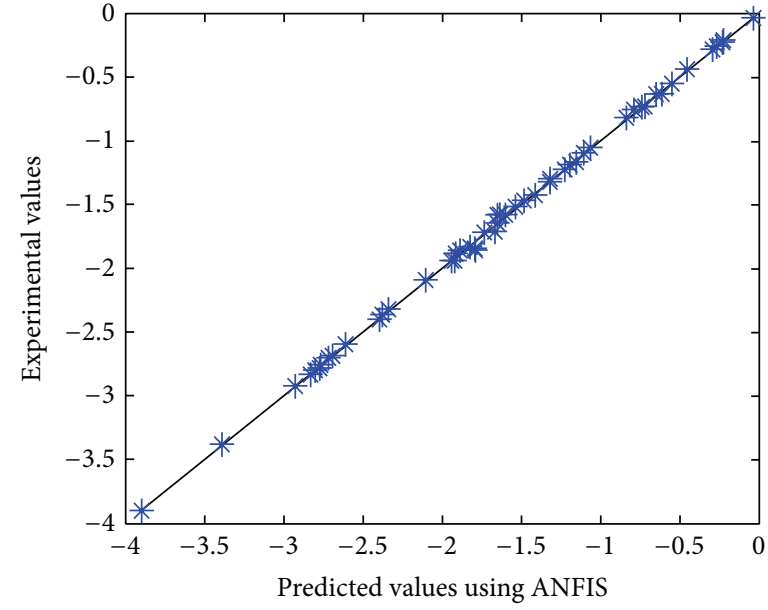

(a)

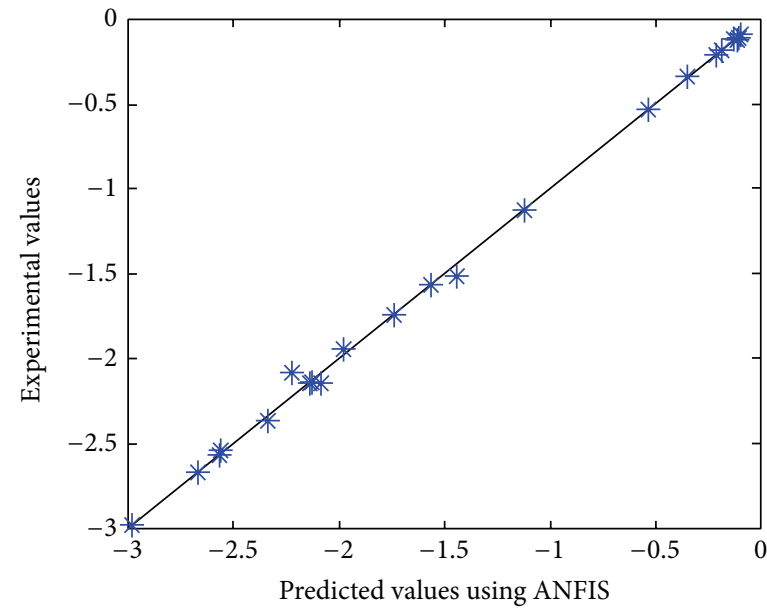

(b)

FIgURE 7: Comparison between the experimental and interpreted results for (a) training data and (b) testing data.

TABLE 2: The comparison between experimental and interpreted results for testing data.

\begin{tabular}{|c|c|c|c|c|c|c|}
\hline \multirow{2}{*}{ Voltage } & \multirow{2}{*}{ Pressure } & \multirow{2}{*}{$\Phi_{1}$} & \multirow{2}{*}{$\Phi_{2}$} & \multirow{2}{*}{ Experimental } & \multicolumn{2}{|c|}{ Hard X-ray $\left(\mathrm{V} \cdot \mathrm{s} \times 10^{-7}\right)$} \\
\hline & & & & & SD & ANFIS \\
\hline 11 & 2.5 & 90 & 90 & -0.104 & 0.01 & -0.11022 \\
\hline 11 & 4 & 90 & 90 & -1.562 & 0.21 & -1.56031 \\
\hline 11 & 6.5 & 90 & 90 & -0.212 & 0.08 & -0.21855 \\
\hline 12 & 3 & 90 & 90 & -0.098 & 0.01 & -0.09225 \\
\hline 12 & 7 & 90 & 90 & -0.126 & 0.06 & -0.12429 \\
\hline 13 & 4 & 90 & 90 & -0.352 & 0.106 & -0.33832 \\
\hline 13 & 5.5 & 90 & 90 & -1.44 & 0.167 & -1.51299 \\
\hline 11 & 2 & 90 & 84.28 & -0.108 & 0.026 & -0.10941 \\
\hline 11 & 4 & 90 & 84.28 & -1.124 & 0.063 & -1.12227 \\
\hline 11 & 5 & 90 & 84.28 & -2.141 & 0.218 & -2.14826 \\
\hline 12 & 3 & 90 & 84.28 & -0.187 & 0.063 & -0.17701 \\
\hline 12 & 4.5 & 90 & 84.28 & -1.743 & 0.191 & -1.742 \\
\hline 13 & 3.5 & 90 & 84.28 & -0.535 & 0.196 & -0.53538 \\
\hline 13 & 6 & 90 & 84.28 & -2.67 & 0.158 & -2.67017 \\
\hline 11 & 2.5 & 79.7 & 79.7 & -0.114 & 0.017 & -0.11976 \\
\hline 11 & 4.5 & 79.7 & 79.7 & -2.57 & 0.183 & -2.56853 \\
\hline 11 & 5.5 & 79.7 & 79.7 & -2.09 & 0.091 & -2.14214 \\
\hline 12 & 3.5 & 79.7 & 79.7 & -1.98 & 0.0416 & -1.94411 \\
\hline 12 & 5.5 & 79.7 & 79.7 & -2.34 & 0.0231 & -2.36587 \\
\hline 12 & 6 & 79.7 & 79.7 & -2.22 & 0.02 & -2.08493 \\
\hline 13 & 3.5 & 79.7 & 79.7 & -2.56 & 0.116 & -2.54087 \\
\hline 13 & 5 & 79.7 & 79.7 & -2.98 & 0.0354 & -2.97635 \\
\hline 13 & 6 & 79.7 & 79.7 & -2.13 & 0.0495 & -2.13296 \\
\hline
\end{tabular}

SD stands for standard deviation for each experimental measurement.

have been recorded in the laboratory. On the other hand, the interpreted values are those which have been generated by the ANFIS network. Each correspondent pair of these two values produces a blue star which can be seen in the diagram. More alighnment of stars with the regrassion diagram $(y=$ $x$ ), denotes more precision of calculations. According to the definition, the network errors are described by difference between experimental and interpreted values or distance from the regression diagram $(y=x)$. As Figure 7 shows, the proposed ANFIS network has achieved good agreement with the experimental results.

Table 2 shows the comparison between experimental and interpreted results for testing data. Table 3 shows the obtained errors for the ANFIS, where the mean relative 


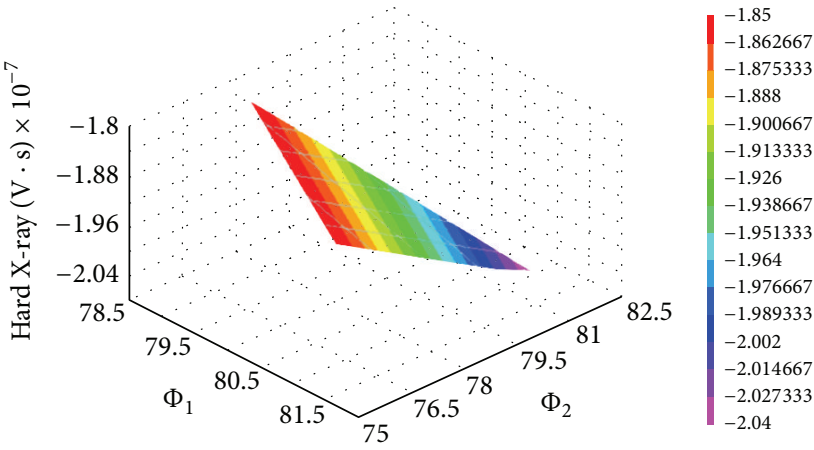

(a)

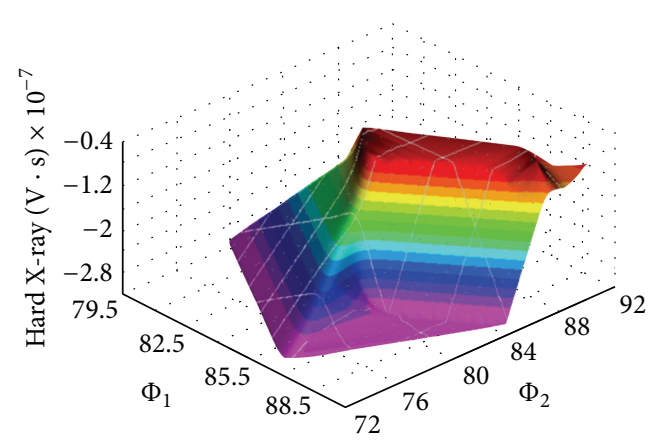

(c)

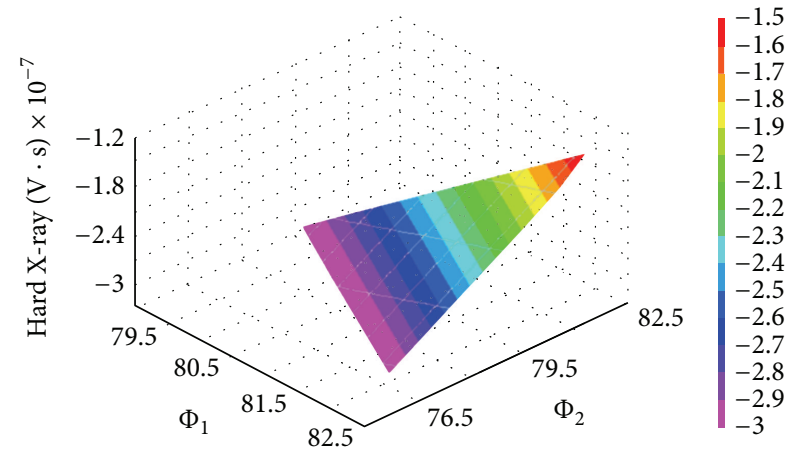

(b)

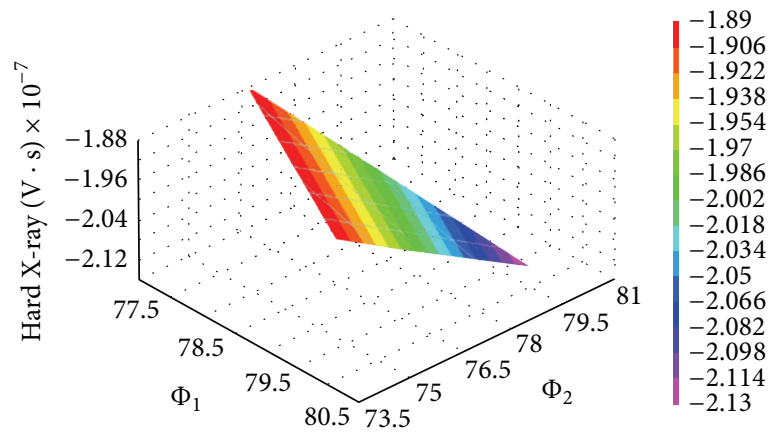

(d)

FIGURE 8: The obtained HXR emission from different anode shapes using the proposed ANFIS network by $V=13 \mathrm{kV}, 70^{\circ}<\Phi_{1}$ and $\Phi_{2}<90^{\circ}$, $\Phi_{2}<\Phi_{1}$, and (a) $P=3$ mbar, (b) $P=4$ mbar, (c) $P=5$ mbar, and (d) $P=6$ mbar.

TABLE 3: Obtained errors for training and testing results of the ANFIS.

\begin{tabular}{lcc}
\hline Error & Train & Data \\
& 1.12 & Test \\
\hline MRE \% & 0.013 & 2.18 \\
MAE & 0.020 & 0.017 \\
RMSE & 0.99972 & 0.035 \\
CF & & 0.99938 \\
\hline
\end{tabular}

error percentage (MRE\%), the mean absolute error (MAE), the root mean square error (RMSE), and the correlation factor (CF) of the ANFIS are calculated by (7), where $N$ is the number of data and " $X(\operatorname{Exp})$ " and " $X($ Intp)" stand for experimental and interpreted values, respectively:

$$
\begin{gathered}
\mathrm{MRE} \%=100 \times \frac{1}{N} \sum_{i=1}^{N}\left|\frac{X_{i}(\operatorname{Exp})-X_{i}(\text { Intp })}{X_{i}(\operatorname{Exp})}\right|, \\
\mathrm{RMSE}=\left[\frac{\sum_{i=1}^{N}\left(X_{i}(\operatorname{Exp})-X_{i}(\operatorname{Intp})\right)^{2}}{N}\right]^{0.5}, \\
\mathrm{CF}=1-\left[\frac{\sum_{i=1}^{N}\left(X_{i}(\operatorname{Exp})-X_{i}(\text { Intp })\right)^{2}}{\sum_{i=1}^{N}\left(X_{i}(\operatorname{Exp})\right)^{2}}\right], \\
\mathrm{MAE} \%=100 \times \frac{1}{N} \sum_{i=1}^{Z} \mid X_{i}(\operatorname{Exp})-X_{i}(\text { Intp }) \mid .
\end{gathered}
$$

Examining Table 2 and Figure 7 indicates the high proximity between interpreted and experimental results for HXR radiation which assure the capability of proposed ANFIS network as an accurate, reliable method to interpret radiated HXR in PF devices.

To find out the best configuration in each pressure, Figure 8 is presented in which pressure varies from 3 up to 6 mbar, respectively, while the HXR values are calculated by ANFIS network. Hence, the maximum HXR intensities are derived to be $-2.04 \times 10^{-7},-3 \times 10^{-7},-2.85 \times 10^{-7}$, and $-2.13 \times 10^{-7}(\mathrm{~V} \cdot \mathrm{s})$, respectively, in $\left(\Phi_{2}, \Phi_{1}\right)=(81.3,81.2),(79.3$, $79.2),(87.1,73.2)$, and $(79.8,79.7)$. From these results, it is clear that ANFIS network can be used as an accurate method to calculate the highest X-ray yield in plasma focus devices.

\section{Conclusion}

This paper proposes an adaptive neurofuzzy inference system to optimize the design of conical-based anode tips in plasma focus devices to achieve the highest $\mathrm{X}$-ray yield. Comparison between experimental and interpreted results using ANFIS verifies the validity of network to determine the best configurations in which the HXR yield is maximized. In this case, the highest yield is calculated in $\left(\Phi_{2}, \Phi_{1}\right)=(79.3,79.2)$ of anode configuration at pressure of 4 mbar. Moreover, the application of this simulation can be tested in other cases in which plasma foci are used as electron, ion, or neutron source by defining related output parameter and testing finite experimental data. 


\section{Conflict of Interests}

The authors declare that there is no conflict of interests regarding the publication of this paper.

\section{References}

[1] R. Lebert, W. Neff, and D. Rothweiler, "Pinch plasma source for $\mathrm{X}$-ray microscopy with nanosecond exposure time," Journal of X-Ray Science and Technology, vol. 6, no. 2, pp. 107-140, 1996.

[2] R. R. Prasad, M. Krishnan, J. Mangano, P. A. Greene, and N. Qi, "Neon dense plasma focus point X-ray source for 0.25 um lithography," in Electron-Beam, X-Ray, and Ion-Beam Submicrometer Lithographies for Manufacturing IV, vol. 2194 of Proceedings of SPIE, pp. 120-128, March 1994.

[3] M. Sohrabi, M. Habibi, G. H. Roshani, and V. Ramezani, "A novel method for observation by unaided eyes of nitrogen ion tracks and angular distribution in a plasma focus device using $50 \mathrm{~Hz}-\mathrm{HV}$ electrochemically-etched polycarbonate detectors," Radiation Measurements, vol. 47, no. 7, pp. 530-536, 2012.

[4] W. L. Harries, J. H. Lee, and D. R. McFarland, "Trajectories of high energy electrons in a plasma focus," Plasma Physics, vol. 20, no. 2, pp. 95-106, 1978.

[5] H. Asai and I. Veno, "Neutron source based on the repetitive dense plasma focus model," Fusion Engineering and Design, vol. 7, pp. 335-343, 1989.

[6] G. R. Neil and R. S. Post, "Observation of overdense infrared scattering from a post pinch plasma focus," Plasma Physics, vol. 23, no. 5, pp. 425-434, 1981.

[7] I. V. Volobuev, V. A. Gribkov, D. Denus et al., "plasma-focus devices," Soviet Journal of Plasma Physics, vol. 14, pp. 401-405, 1988.

[8] J. N. Feugeas, E. C. Llonch, C. O. De González, and G. Galambos, "Nitrogen implantation of AISI 304 stainless steel with a coaxial plasma gun," Journal of Applied Physics, vol. 64, no. 5, pp. 2648-2651, 1988.

[9] H. Kelly, A. Lepone, A. Márquez, D. Lamas, and C. Oviedo, "Coating on metallic samples produced by a small energy plasma focus," Plasma Sources Science and Technology, vol. 5, no. 4, pp. 704-709, 1996.

[10] J. Pouzo, M. Milanese, and R. Moroso, "Portable neutron probe for soil humidity measurements," AIP Conference Proceedings, vol. 669, no. 1, pp. 277-280, 2003.

[11] C. Moreno, A. Clausse, J. Martínez et al., "Small-chamber 4.7 kJ plasma focus for applications," AIP Conference Proceedings, vol. 563, no. 1, pp. 276-281, 2001.

[12] S. Lee, P. Lee, G. Zhang et al., "High rep rate high performance plasma focus as a powerful radiation source," IEEE Transactions on Plasma Science, vol. 26, no. 4, pp. 1119-1126, 1998.

[13] P. Lee, X. Feng, G. X. Zhang, M. H. Liu, and S. Lee, "Electron lithography using a compact plasma focus," Plasma Sources Science and Technology, vol. 6, no. 3, pp. 343-348, 1997.

[14] E. P. Bogolyubov, V. D. Bochkov, V. A. Veretennikov et al., "A powerful soft X-ray source for X-ray lithography based on plasma focusing," Physica Scripta, vol. 57, no. 4, pp. 488-494, 1998.

[15] F. N. Beg, I. Ross, A. Lorenz, J. F. Worley, A. E. Dangor, and M. G. Haines, "Study of X-ray emission from a table top plasma focus and its application as an X-ray backlighter," Journal of Applied Physics, vol. 88, no. 6, pp. 3225-3230, 2000.
[16] R. Petr, A. Bykanov, J. Freshman et al., "Performance summary on a high power dense plasma focus X-ray lithography point source producing $70 \mathrm{~nm}$ line features in AlGaAs microcircuits," Review of Scientific Instruments, vol. 75, no. 8, pp. 2551-2559, 2004.

[17] V. A. Gribkov, A. Srivastava, P. L. C. Keat, V. Kudryashov, and P. Lee, "Operation of NX2 dense plasma focus device with argon filling as a possible radiation source for micro-machining," IEEE Transactions on Plasma Science, vol. 30, no. 3, pp. 1331-1338, 2003.

[18] F. Castillo-Mejía, M. M. Milanese, R. L. Moroso, J. O. Pouzo, and M. A. Santiago, "Small plasma focus studied as a source of hard X-ray," IEEE Transactions on Plasma Science, vol. 29, no. 6, pp. 921-926, 2001.

[19] F. Castillo, J. J. E. Herrera, J. Rangel et al., "Neutron anisotropy and X-ray production of the FN-II dense plasma focus device," Brazilian Journal of Physics, vol. 32, no. 1, pp. 3-12, 2002.

[20] S. Hussain, M. Shafiq, R. Ahmad, A. Waheed, and M. Zakaullah, "Plasma focus as a possible X-ray source for radiography," Plasma Sources Science and Technology, vol. 14, no. 1, pp. 61-69, 2005.

[21] R. S. Rawat, "High energy density pulsed plasmas in plasma focus: novel plasma processing tool for nanophase hard magnetic material synthesis," Nanoscience and Nanotechnology Letters, vol. 4, no. 3, pp. 251-274, 2012.

[22] S. Hussain, S. Ahmad, M. Z. Khan, M. Zakaullah, and A. Waheed, "Plasma focus as a high intensity flash X-ray source for biological radiography," Journal of Fusion Energy, vol. 22, no. 3, pp. 195-200, 2003.

[23] V. Raspa, L. Sigaut, R. Llovera et al., "Plasma focus as a powerful hard X-ray source for ultrafast imaging of moving metallic objects," Brazilian Journal of Physics, vol. 34, no. 4B, pp. 16961699, 2004.

[24] N. V. Filippov, T. I. Filippova, and V. P. Vinogradov, "Densa high-temperature plasma in a non-clyndrical Z-pinch compression," Supplement to the Journal Nuclear Fusion-IAEA, vol. 2, article 577, 1962.

[25] J. W. Mather, "Formation of a high-density deuterium plasma focus," Physics of Fluids, vol. 8, no. 2, pp. 336-377, 1965.

[26] D. J. Johnson, "Study of the X-ray production mechanism of a dense plasma focus," Journal of Applied Physics, vol. 45, pp. 1147$1153,1974$.

[27] M. Zakaullah, I. Ahmad, A. Omar, G. Murtaza, and M. M. Beg, "Effects of anode shape on plasma focus operation with argon," Plasma Sources Science and Technology, vol. 5, no. 3, pp. 544552, 1996.

[28] M. Mahtab and M. Habibi, "Nitrogen soft and Hard X ray emissions using different shapes of anodes in a $4 \mathrm{~kJ}$ Plasma focus device of Anodes in a $4 \mathrm{~kJ}$ plasma focus device," Plasma Physics Reports, vol. 40, no. 1, pp. 34-39, 2014.

[29] M. Habibi, R. Amrollahi, M. Attaran, and R. Etaati, "Design, construction and the first experiments on the Amirkabir plasma focus (APF) facility," Plasma Devices and Operations, vol. 16, no. 3, pp. 163-169, 2008.

[30] R. Baghdadi, R. Amrollahi, M. Habibi, and G. R. Etaati, "Investigation of the neutron angular distribution and neutron yield on the apf plasma focus device," Journal of Fusion Energy, vol. 30, no. 1, pp. 72-77, 2011.

[31] J. S. R. Jang, C. T. Sun, and E. Mizutani, "Neuro-fuzzy and soft computing a computational approach to learning and machine intelligence," IEEE Transactions on Automatic Control, vol. 42, no. 10, pp. 1482-1484, 1997. 
[32] J.-S. R. Jang and C.-T. Sun, "Neuro-fuzzy modeling and control," Proceedings of the IEEE, vol. 83, no. 3, pp. 378-406, 1995.

[33] H. Bunke and A. Kandel, Neruro-Fuzzy Pattern Recognition, vol. 23, Chapters 2-3, World Scientific, Singapore, 2000. 

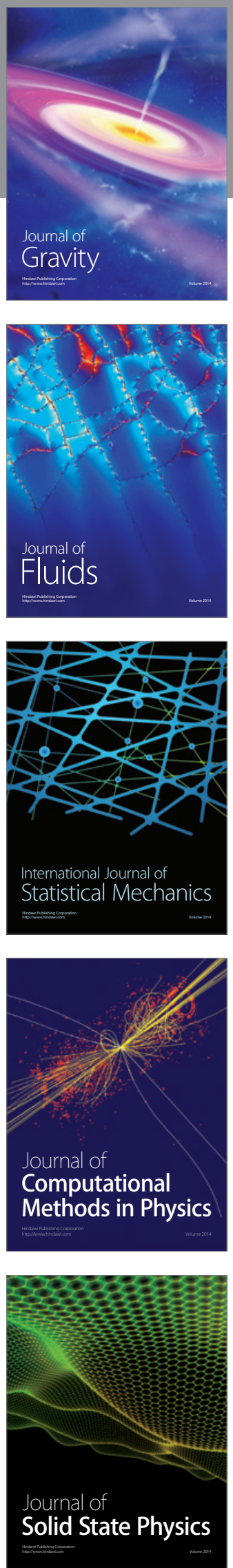

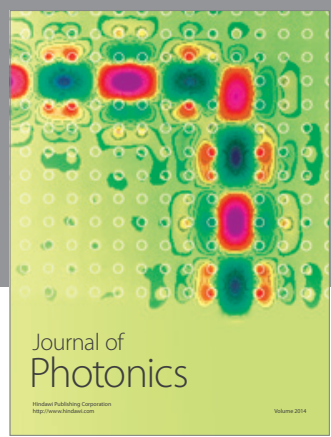

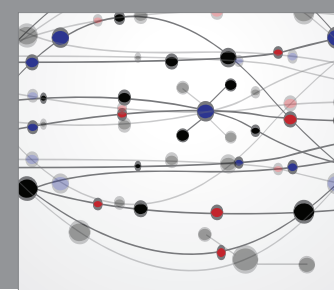

The Scientific World Journal

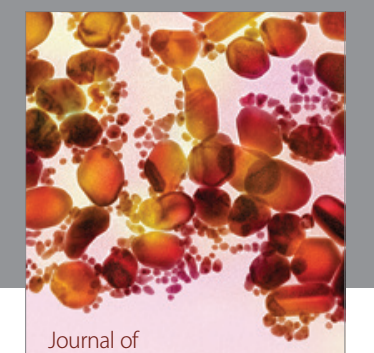

Soft Matter
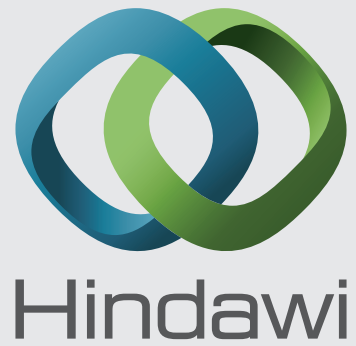

Submit your manuscripts at

http://www.hindawi.com
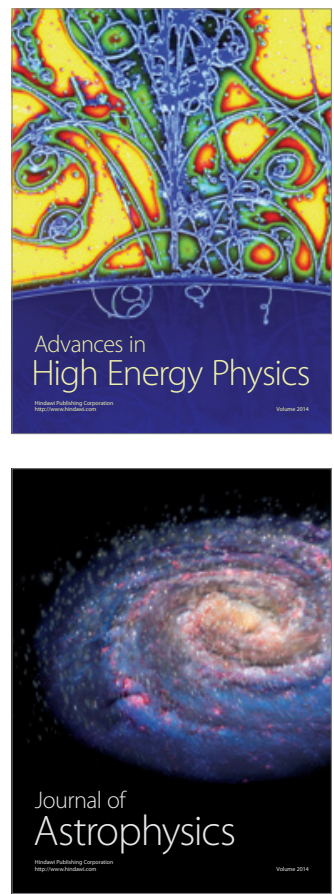
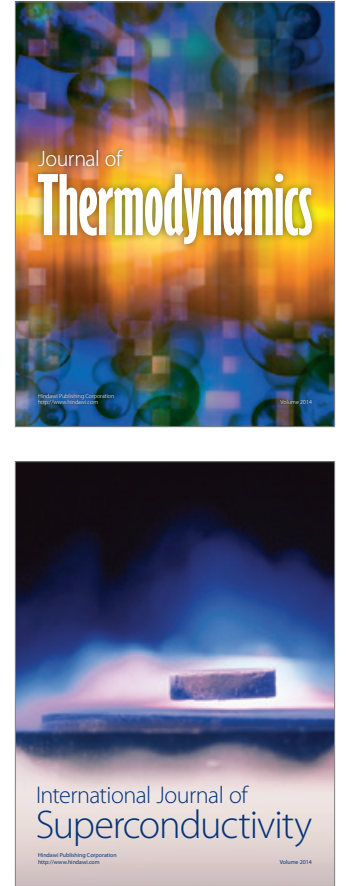
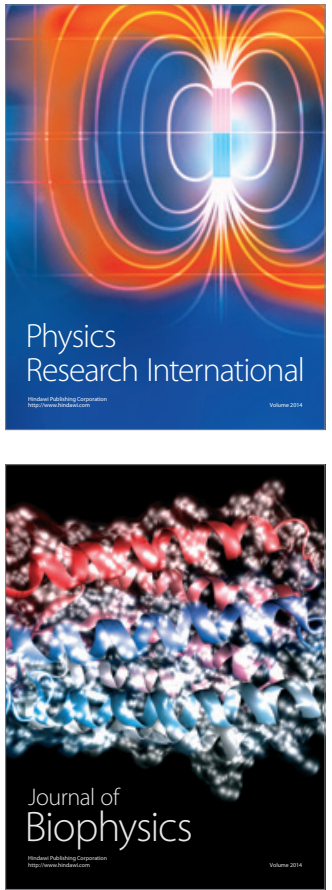
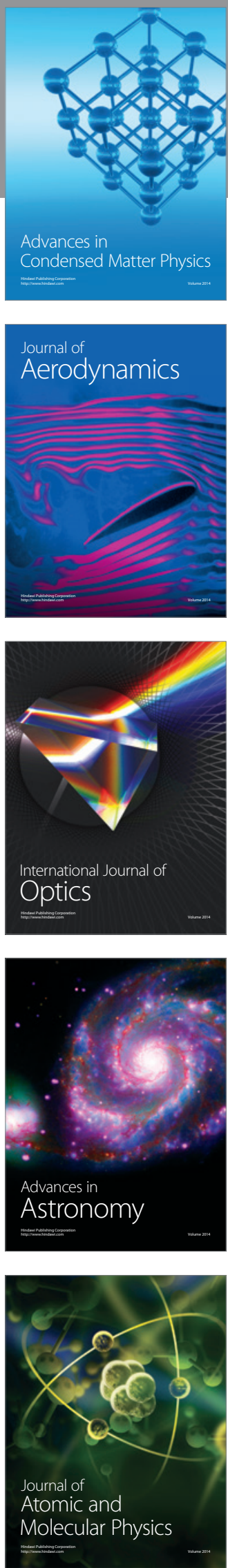